\title{
Les confréries de dévotion traditionnelles et le réveil religieux à Montréal au XIX ${ }^{\mathrm{e}}$ siècle
}

\section{Brigitte Caulier}

Volume 53, 1986

Le renouveau religieux à Montréal au XIX ${ }^{\mathrm{e}}$ siècle

URI : https://id.erudit.org/iderudit/1006968ar

DOI : https://doi.org/10.7202/1006968ar

Aller au sommaire du numéro

\section{Éditeur(s)}

Les Éditions Historia Ecclesiæ Catholicæ Canadensis Inc.

ISSN

0318-6172 (imprimé)

1927-7067 (numérique)

Découvrir la revue

Citer cet article

Caulier, B. (1986). Les confréries de dévotion traditionnelles et le réveil religieux à Montréal au XIX ${ }^{\mathrm{e}}$ siècle. Sessions d'étude - Société canadienne d'histoire de l'Église catholique, 53, 23-40. https://doi.org/10.7202/1006968ar
Résumé de l'article

Les confréries de dévotion montréalaises, fondées sous le Régime français, comme la Sainte-Famille, la Congrégation des hommes de Ville-Marie ainsi que l'Adoration perpétuelle du Saint-Sacrement et de la Bonne-Mort, ont vu les notables laïques retourner vers elles avant les années 1840, période du réveil religieux. Pendant cette dernière, le recrutement connaît une accélération. Dans la première moitié du siècle, les Sulpiciens, très attachés à cette forme d'encadrement des laïcs, ont restauré les règles et usages anciens, tout en affermissant leurs prérogatives de directeurs de ces confréries, surtout chez les dames de la Sainte-Famille et à la Bonne-Mort (à majorité féminine). Outre la discipline, le clergé a rétabli ces associations comme écoles de piété exigeante, à la fois sur le plan individuel et collectif. Les solennités sont devenues plus nombreuses et les associés les célébrèrent avec un faste accru, traduisant le goût nouveau de la pompe romaine. Ils tiraient fierté d'appartenir à ces anciennes confréries et donnaient tout à la fois, l'exemple au reste des paroissiens de Notre-Dame de Montréal.
Tous droits réservés @ Les Éditions Historia Ecclesiæ Catholicæ Canadensis Inc., 1986
Ce document est protégé par la loi sur le droit d'auteur. L'utilisation des services d'Érudit (y compris la reproduction) est assujettie à sa politique d'utilisation que vous pouvez consulter en ligne.

https://apropos.erudit.org/fr/usagers/politique-dutilisation/ 


\title{
Les confréries de dévotion traditionnelles et le réveil religieux à Montréal au XIXe siècle*
}

\author{
Brigitte CAulier \\ Département d'histoire \\ Université Laval
}

RÉSUMÉ

Les confréries de dévotion montréalaises, fondées sous le Régime français, comme la Sainte-Famille, la Congrégation des hommes de VilleMarie ainsi que l'Adoration perpétuelle du Saint-Sacrement et de la BonneMort, ont vu les notables laïques retourner vers elles avant les années 1840, période du réveil religieux. Pendant cette dernière, le recrutement connaît une accélération. Dans la première moitié du siècle, les Sulpiciens, très attachés à cette forme d'encadrement des laïcs, ont restauré les règles et usages anciens, tout en affermissant leurs prérogatives de directeurs de ces confréries, surtout chez les dames de la Sainte-Famille et à la Bonne-Mort (à majorité féminine). Outre la discipline, le clergé a rétabli ces associations comme écoles de piété exigeante, à la fois sur le plan individuel et collectif. Les solennités sont devenues plus nombreuses et les associés les célébrèrent avec un faste accru, traduisant le goût nouveau de la pompe romaine. Ils tiraient fierté d'appartenir à ces anciennes confréries et donnaient tout à la fois, l'exemple au reste des paroissiens de Notre-Dame de Montréal.

Le clergé montréalais, depuis les débuts de la colonie, s'est servi des confréries de dévotion afin d'encadrer les laïcs dans leur piété et leur conduite morale. Certaines de ces associations volontaires ont traversé les siècles pour s'éteindre dans les années 1960. La confrérie des dames de la Sainte-Famille, dont les prémices montréalais remontent à 1663 , et la

* Cette étude, menée dans le cadre d'un doctorat, a été financée par le Conseil de recherches en sciences humaines du Canada. 
Congrégation des hommes de Ville-Marie, rattachée à la Prima Primaria romaine en 1693, sont de celles-là. Tandis que l'Adoration perpétuelle du Saint-Sacrement et de la Bonne-Mort, fondée en 1732, a disparu au début $\mathrm{du} \mathrm{XX}^{\mathrm{e}}$ siècle.

La simple évolution du recrutement de ces confréries révèle, à la fois, la capacité d'encadrement du clergé et l'intérêt ou la désaffection des laïcs adultes vis-à-vis de ces organisations. Aussi, nous permet-elle d'appréhender l'impact du réveil religieux des années 1840, tout en fournissant des précisions sur sa chronologie: est-il sensible un peu avant 1840 , les confréries ayant alors eu un rôle moteur dans le changement de mentalité, par l'exemple offert aux autres laïcs; ou bien ont-elles simplement accompagné les changements, servant de structure d'accueil à une nouvelle ferveur, ou enfin, faut-il attendre quelques années après la date clé de la prédication de $\mathrm{M}^{\mathrm{gr}}$ de Forbin-Janson pour que les confréries réagissent?

\section{1. Évolution du recrutement entre 1800 et $\mathbf{1 8 5 0}$}

Après avoir connu des difficultés de recrutement dans la seconde moitié du XVIII e siècle et au début du $\mathrm{XIX}^{\mathrm{e}}$, les confréries de dévotion montréalaises ont progressivement admis de nouveaux membres selon une périodisation différenciée pour chacune (voir le graphique comparatif).

Dans les deux premières décennies du siècle, la confrérie de la BonneMort (suivant l'abréviation courante depuis sa fondation) accueille respectivement 126 et 124 personnes, ce qui représente une légère amélioration par rapport au second XVIII siècle. La reprise décisive intervient dans les années 1820 où l'effectif nouvellement reçu progresse de $48 \%$ par rapport à la décennie antérieure. La limite des 200 nouvelles admissions franchie le restera jusqu'à la fin des années 1880 . L'inversement de la tendance s'inscrit donc pour cette confrérie avant 1840. Entre 1825 et 1839, 20 personnes et plus entrent annuellement (à trois années près); elles sont proches des 40 en 1835. À partir de cette date, il s'agit d'un minimum, car la tenue plus aléatoire des livres de contributions par rapport au registre des réceptions nous a fait rejeter 44 recrues pour lesquelles la date d'entrée est incertaine entre 1820 et 1839 . Un nouveau seuil est franchi à partir des années 1840 où le recrutement fait un bond à 333 associés et à 488 si nous y joignons les inscriptions à date imprécise. Nous serions donc en face d'un doublement par rapport aux dix ans précédents. À partir de là, la progression se poursuit avec, dans les années 1850, 372 personnes qui intègrent la Bonne-Mort et 495 si nous retenons les dates incertaines. La courbe annuelle des entrées révèle encore des fluctuations importantes entre 1840 et 1849 , avec deux minima en 1840 et 1846 , tandis que dans les années 1850 les écarts s'atténuent. 
Cette reprise du recrutement s'accompagne de mutations profondes sur le plan du dimorphisme sexuel. Depuis sa fondation, la Bonne-Mort a toujours admis majoritairement des femmes. Toutefois, le déclin de la présence masculine s'accélère à partir des années 1830 et devient définitif dans les années 1850 , où les femmes monopolisent à plus de $90 \%$ les entrées de la Bonne-Mort. De $22 \%$ entre 1800 et 1809 , les hommes ne représentent plus que $5 \%$ entre 1850 et 1859 . La concurrence d'une autre confrérie de dévotion semblable ne peut expliquer ce phénomène, puisque l'Adoration nocturne n'apparaît qu'en 1881. Cette féminisation n'a rien d'exceptionnel, si nous la comparons à ce qui s'est passé en Europe où, par exemple, la confrérie de l'Assomption de Saumur ne recrute plus que des femmes à quelques exceptions près au XIXe siècle ${ }^{1}$.

L'analyse du statut civil des consœurs à leur réception apporte d'autres éléments explicatifs à ces changements. Depuis sa fondation et jusque dans les années 1830, la Bonne-Mort a reçu en majorité des femmes mariées et des veuves. Or, à partir de 1840 , le rapport s'inverse et elle accueille essentiellement des célibataires, avec un écart qui ira en s'accentuant dans la seconde moitié du siècle. De plus, le sous-groupe des veuves augmente fortement dès la première décennie de celui-ci, frôlant $20 \%$ des entrées féminines. Nous assistons donc à un vieillissement du recrutement qui touche des femmes ṕlus libres de leur temps pour la dévotion, et plus sensibles à la nécessité de se préparer à la mort, par le décès de leurs proches. En contre partie, apparaît le détachement relatif des mères de famille sur lesquelles le clergé avait toujours compté pour entretenir la piété auprès de leur mari et des enfants. En effet, un sondage effectué sur un échantillon nominal (nom des consœurs commençant par A, B, C, D, G) révèle une moyenne de 15 années de mariage à leur réception entre 1800 et 1849 .

Du côté des célibataires, les effectifs bénéficient de l'inscription des religieuses, sœurs grises pour la plupart, surtout à partir des années 1820 , où elles représentent alors $11 \%$ des recrues féminines (contre 5\% ou moins au $\mathrm{XIX}^{\mathrm{e}}$ ), tandis qu'elles plafonneront dans la décennie 1880 à $32 \%$. Elles ont donc soutenu le recrutement massif du XIXe siècle, montrant ainsi l'exemple aux laïcs, en s'inscrivant à la Bonne-Mort. Leur chapelain et confesseur, sulpicien, a certainement encouragé leur adhésion à la confrérie qui proposait une dévotion valorisée dans les milieux religieux: le saint Sacrement. Les adhésions de femmes célibataires ont fait un bond dès les dernières années du siècle précédent (de $23 \%$ à $44 \%$ ) et se maintiennent au-dessus de $32 \%$ pour atteindre $47 \%$ du total des nouvelles consœurs entre 1840 et 1849 . Contrairement au Régime français où des jeunes filles entraient à la confrérie

1 F. Poirault, "La confrérie de l'Assomption de Saumur, 1402-1903", Annales de Bretagne et des pays de l'Ouest (1979), p. 424. 
peu avant leur mariage, les célibataires recrutées au XIXe garderont ce statut définitivement. Or, la qualité des sources n'est pas en cause ici, car les changements d'état civil étaient bien enregistrés dans les catalogues, les directeurs ajoutant simplement le nom de l'époux qui n'a jamais fait disparaître le nom de jeune fille pendant ces deux siècles. La Bonne-Mort a donc accueilli au XIXe un grand nombre de célibataires dont la situation en ville demeurait très précaire et qui, pour éviter la solitude dans leurs derniers instants, choisirent d'adhérer à cette confrérie. Jean-Claude Robert a montré que la ville de Montréal a connu un déséquilibre démographique où les femmes étaient beaucoup plus nombreuses que les hommes «surtout à partir de $1844 »^{2}$.

Ce facteur, condamnant une partie des Montréalaises au célibat, a certainement amplifié la féminisation de la Bonne-Mort au XIXe siècle par rapport à celui, purement religieux, qui intervient en Europe où les activités de dévotion deviennent affaires de femmes. Cette féminisation s'est accompagnée d'un vieillissement des recrues, qui transparaît dans l'établissement d'un système de cotisations proportionnelles et majorées avec l'âge, comme les polices d'assurances: "Après la réception, suivre le règlement pour la souscription d'entrée, cad exiger, au lieu de $25 \mathrm{cts}$, 1\# après 30 ans, 2\# après 40 et ainsi de suite, à moins de bonnes raisons» ${ }^{3}$.

Première association fondée pour les adultes, la Sainte-Famille a connu un recrutement exclusivement féminin, dès les premières années à Montréal, contrairement à Québec où $\mathrm{M}^{\mathrm{gr}}$ de Laval fit une entorse à son propre règlement. De même, rares furent les célibataires montréalaises qui intégrèrent cette association.

La confrérie a très mal supporté la fin du XVIII ${ }^{e}$ siècle et la stagnation des admissions à un niveau infime (16 dames entre 1790 et 99 , puis entre 1800 et 1809) se poursuit jusqu'en 1819 avec plusieurs années sans aucune entrée, notamment entre 1789 et 1794, ou bien avec une seule inscription, dans les années 1800 et 1810 . Le réveil survient brusquement à la décennie suivante où le nouvel apport de 68 consœurs renvoie aux chiffres obtenus sous le Régime français, les dix années qui lui succèdent confirmant l'élan. La courbe annuelle, néanmoins, révèle une ascension constante dans les années 1820 suivie d'une retombée au début des années 1830 , vite corrigée malgré tout. C'est dans les années 1840 que se fait le décollage des admissions qui doublent à ce moment et particulièrement à partir de 1845 .

2 Jean-Claude Robert, Montréal 1821-1871, aspects de l'urbanisation, Thèse de Ph.D., EHESS, 1977.

3 Cahier de comptes de la confrérie de la Bonne-Mort, 1874-1886, Archives de la compagnie de Saint-Sulpice de Montréal (désormais ACSSM), S.35, 16.10. Nous conserverons l'orthographe des auteurs sans en signaler les irrégularités. 
Cette année-là, 27 femmes font leur entrée dans la confrérie. Au total, 145 femmes y ont adhéré entre 1840 et 49 et 123 entre 1850 et 59.

Contrairement à la Bonne-Mort, nous disposons d'une estimation des effectifs, précise lorsque les directeurs les mentionnent ou bien approchée grâce aux listes de contributions. Nous obtenons ainsi des clichés successifs du nombre total de femmes regroupées dans la confrérie, et susceptibles de participer aux exercices de piété. Dans les premières années du XIXe siècle, les cotisations se maintiennent péniblement au-dessus de 25 associées et confirment l'absence de recrutement. Ce marasme n'a pas pour origine l'absentéisme, mais bien le manque de dynamisme et d'attrait de la confrérie. Les effectifs réels issus des catalogues et établis par les directeurs en 1816, 1817 et 1818, révèlent que 34 dames appartenaient à la Sainte-Famille dans les deux premières années; en 1818, 46 étaient dénombrées, différence explicable par un rappel des brebis égarées. Les effectifs progressent véritablement à partir de 1826 où ils doublent, sans rattraper néanmoins les niveaux des années 1760-1767 avant 1828, tandis que les années suivantes marquent un temps de consolidation de ce nouvel acquis qui place l'effectif au-dessus d'une centaine de consœurs. Celui-ci prend son essor dans les années 1840 avec une moyenne de 171 personnes. Vingt ans plus tard, 250 mères de famille appartiennent à la confrérie et en 1886, 880 en sont membres. Malgré cet accroissement spectaculaire qui s'est poursuivi dans la seconde moitié du siècle, les effectifs n'ont pas suivi la progression démographique et le fort mouvement d'urbanisation qui fait presque doubler la population de la ville entre 1852 et 1871 .

En considérant le type de recrutement, en fonction de l'impact réel qu'il pouvait avoir à l'intérieur de chaque famille, les résultats sont alors meilleurs. En rejoignant les mères qui devaient servir de modèle à leur entourage, la confrérie touchait beaucoup plus de personnes que ne laissent paraitre les chiffres, encore faut-il faire intervenir le facteur de l'âge puisqu'une mère en pleine activité a peut-être plus à transmettre qu'une femme qui a déjà marié ses enfants et qui retrouve ses amies aux réunions chaque quinzaine. Le recrutement chez les veuves n'a guère touché la confrérie depuis sa fondation jusqu'à la fin du XIXe siècle. Entre 1820 et 1859, les femmes admises à la Sainte-Famille avaient 13 ans de mariage derrière elles, à leur entrée. Par la suite, la demande des personnes âgées s'accentue, si l'on en croit les normes d'admission édictées par M. Martineau, directeur de la confrérie de 1866 à 1887, qui rejette les femmes de plus de 60 ans. L'explosion des effectifs au XIXe siècle ne doit donc pas faire illusion: elle n'a pas entraîné de passage entre les confréries réservées aux jeunes filles vers la Sainte-Famille (8 cas au $\mathrm{XIX}$ ). L'encadrement féminin s'est donc toujours relâché dans les premières années du mariage où les femmes sont accaparées par leurs tâches ménagères. Le retour des dames à la confrérie s'est opéré avant les années 
1840 et a été fortement encouragé par M. Sattin, nommé à sa tête en 1825. L'ampleur de ses efforts nous est bien connue grâce au cahier qu'il tenait régulièrement pour ne rien oublier des consignes à transmettre aux dames. Ce directeur est ainsi à l'origine d'une tradition séculaire qui offre à l'historien un témoignage unique sur la vie intime d'une confrérie.

Les archives de la Congrégation des hommes de Ville-Marie se partagent entre les Jésuites et les Sulpiciens qui en reprirent la direction à la mort du père Well en 1917. La reconstitution du recrutement demeure possible à partir de 1768 et pour tout le XIXe siècle avec, toutefois, une marge d'incertitude avant 1804 car nous avons eu recours au livre des recettes de 1768 à 1804 qui n'enregistre pas régulièrement les admissions. Après un passage à vide, à la fin du XVIIIe siècle, certainement accentué par cette sous-inscription, la confrérie attire de nouveaux membres surtout à partir de 1805 jusqu'en 1810, et cinq années plus tard, des pics sont enregistrés qui portent à 84 le nombre des admissions dans les années 1810. Un essoufflement lui succède dans les années 1820 (59 réceptions), mais à partir de 1830 , le recrutement double et augmente de $62 \%$ entre 1840 et 1849 (128 et 207 nouveaux congréganistes). $\mathrm{La}$ courbe détaillée fait apparaître une progression assez régulière dans les années 1840 suivie de deux pics en 1849 et 1850 . Les sommets seront atteints entre 1869 et 1875 ; la reprise perceptible dans la première moitié du siècle a donc eu des prolongements durables.

Nous ne disposons pas, pour la période qui nous intéresse, d'une évaluation précise des effectifs, mais les listes de cotisations annuelles révèlent une stabilité autour de 100-150 membres actifs depuis la fin du XVIIIe jusqu'en 1852. Ces résultats, signes d'une bonne résistance de la Congrégation en une période d'attiédissement de la ferveur au tournant du siècle, sont plus inquiétants lors d'une période de recrutement soutenu. Un mécanisme poussé d'élimination des congréganistes moins zélés a entraîné ce maintien, à niveau inférieur, de l'effectif global.

Contrairement aux deux autres confréries, nous n'avons pas recherché le statut civil et l'âge des nouvelles recrues, car les risques d'erreurs entre homonymes - père et fils entre autres - sont très nombreux, tandis que les consœurs des deux autres confréries étaient toujours identifiées sous leurs deux noms. Malgré tout, nous connaissons la limite d'âge inférieure d'admission qui était de 18 ans jusqu'en 1872, donc de jeunes adultes déjà sur le marché du travail pour la plupart. Les plus jeunes recrues venaient du Collège de Montréal ou du Séminaire, où ils avaient participé aux exercices de la congrégation qui leur était réservée. Aussi, pouvaient-ils entrer directement sans période probatoire s'ils possédaient des lettres patentes et 
«un bon certificat du principal» ${ }^{4}$. Si nous nous en tenons aux renseignements donnés à la réception, les admissions provenant d'anciens collégiens sont infimes jusqu'en 1849. Le même phénomène apparaît pour les adultes qui disposaient, de toute façon, de très peu de congrégations ayant survécu à la disparition des Jésuites, comme celle de Boucherville. Dans l'ensemble, la Congrégation a reçu des hommes dans la force de l'âge n'ayant pas de passé de congréganistes. Là encore, cette association ancienne n'a pas suivi la croissance démographique montréalaise au $\mathrm{XIX}^{\mathrm{e}}$ siècle.

L'évolution comparée du recrutement des confréries, au tournant et dans la première moitié du XIXe siècle, en ce qui concerne plus particulièrement les sœurs jumelles que sont la Sainte-Famille et la Congrégation, montre que cette dernière a mieux résisté que l'autre. La reprise du recrutement à la Sainte-Famille s'effectue de façon lente en ce premier XIXe par rapport à la Congrégation. Les dames ne dépassent les hommes que dans les années 1870. Une telle ténacité chez ces derniers a de quoi surprendre; nous les avons vu se détourner de la Bonne-Mort, mais ils n'ont pas tourné le dos à la Congrégation. Or, les témoignages contemporains présentent des hommes moins religieux que les femmes ${ }^{5}$. À Montréal, la Sainte-Famille est exsangue à la fin du XVIIIe siècle et jusque dans les années 1830 .

Seconde constatation majeure, la remontée du recrutement au XIX ${ }^{e}$ siècle a débuté bien avant la prédication spectaculaire de $\mathrm{M}^{\mathrm{gr}}$ de Forbin-Janson. Tandis que Louis Rousseau relève la césure de 1840 dans le comportement pascal des Montréalais ${ }^{6}$, nous distinguons, depuis les années vingt, le réamorçage de la vitalité confraternelle, avec une accélération dans les années 1840, tandis que la décennie 1850-59 marque un léger essouflement du rythme de croissance. Il existait donc à Montréal, pendant une période d'urbanisation intensive, un noyau de laïcs qui a maintenu une activité associative religieuse, au moment où celle-ci aurait pu subir les contre-coups de la dislocation sociale entraînée par les profondes mutations de la ville.

Ces résultats prennent toute leur importance, au regard des classes sociales touchées par les confréries. En vertu de leurs statuts, celles-ci ne devaient pas pratiquer de ségrégation sociale dans le recrutement de leurs membres, seule la bonne tenue morale servant de critère de sélection. Elles exigeaient une cotisation annuelle modique qui était complétée, pour les

4 Usages de M. Chicoisneau, Archives des Jésuites du Canada français (désormais ASJCF), 2274, p. 119-120.

5 Jean-Pierre Wallot, «La religion catholique et les Canadiens au début du XIX $\mathrm{X}^{\mathrm{e}}$ siècle» dans Un Québec qui bougeait, Montréal, Boréal Express, 1973, p. 203.

6 Louis Rousseau, «La conduite pascale dans la région montréalaise 1831-1865", manuscrit, p. 15. 
dépenses courantes, par des quêtes où chacun donnait selon sa générosité ou ses moyens. En pratique, furent exclus dans les trois associations les représentants des métiers dangereux pour la moralité comme les cabaretiers, les aubergistes et tous ceux vendant de l'alcool au détail. Dans la première moitié du XIXe siècle, la Congrégation écarte formellement les représentants des activités les plus modestes. À partir du 25 novembre 1804, les engagés ne purent devenir congréganistes: «Aucun engagé comme domestique au mois ou à l'année, ne pourra être reçu de la congrégation, parce que n'étant pas

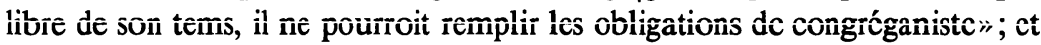
les apprentis uniquement sous condition d' «obtenir la permission du maistre dont il dépent ${ }^{7}$. Cantiniers et charretiers n'étaient pas acceptés non plus à la Congrégation. Outre ces dispositions restrictives, le principal facteur discriminant consiste dans l'obligation de savoir lire pour devenir congréganiste, en raison de leur participation aux offices de la Vierge qui étaient dialogués avec le préfet et ses assistants. En 1850, celui-ci propose «qu'on admette à la congrégation quelques citoyens respectables d'un âge avancé qui ne savent point lire, vû qu'il s'en trouve un certain nombre dans ce cas qui désirent beaucoup d'entrer à la Congrégation ${ }^{8}$. Il ne s'agit donc pas exactement d'une démocratisation comme le laisse clairement présager l'expression "citoyens respectables".

Dans les faits, les trois confréries étudiées ont recruté essentiellement les notables montréalais francophones avec toutes les nuances de fortune possibles. À la Bonne-Mort, artisans et femmes d'artisans l'emportent et plus particulièrement le secteur de la fabrication (entre 24 et $41 \%$ ), avec les boulangers, les cordonniers ainsi que les forgerons. En second, viennent les commerçants et les marchands (1840-49: 9\%), termes qui recouvrent des réalités sociales diverses et peu appréhendables pour l'historien, même si la forte représentation des épiciers nous laisse supposer un recrutement assez modeste. Les professions libérales ne se sont pas tournées vers cette confrérie. Ces tendances nouvelles par rapport au Régime français évoquent un tassement social progressif dans les admissions au XIXe siècle. Toutefois, elles demandent à être nuancées par la forte intégration de célibataires de sexe féminin qu'il est impossible de retracer socialement.

À la Sainte-Famille, les secteurs de la fabrication et du commerce alternent au premier rang des admissions (1840-49: $41 \%$ pour le commerce et $17 \%$ pour la fabrication). Épiciers, marchands de tissus, de chaussures, de peinture et quincailliers ont des femmes qui fréquentent cette confrérie.

7 Registre des délibérations prises dans le conseil de Messieurs les congréganistes de Montréal, 1804-1835, (désormais DC, 1804-1835, ASJCF, 2274, p. 1, 25 novembre 1804.

8 Registre des délibérations du petit conseil (désormais RDpC), 1850-1885, Archives de la fabrique de Notre-Dame de Montréal (désormais AFNDM), 24 avril 1850. 
Contrairement à la Bonne-Mort, les professions libérales ont été représentées (1830-49: autour de 12\%). Toutefois, c'est à partir des années 1860 que se produit un resserrement social très net autour des femmes d'entrepreneurs et de professionnels.

Association réservée aux hommes, la Congrégation a enregistré, assez régulièrement, à partir des années 1820 , le statut professionnel de ses membres. Nous nous sommes contentée de le compléter, dans la mesure du possible. Le secteur économique qui draine le plus de congréganistes au XIXe siècle est le commerce. Excepté entre 1820 et 1829, où il représente moins de $20 \%$, mais avec un taux d'identification de $39 \%$, le commerce rassemble entre 37 et $40 \%$ des admissions jusque dans les années 1850 . Il est suivi de la fabrication qui fournit plus du quart des nouvelles recrues entre 1830 et 1859, tandis que la construction rassemble autour de $16 \%$ des congréganistes. Les professions libérales se sont détournées de la Congrégation dans les années 1840-50 ( 5 et 3,5\%), pour y revenir en nombre dans les années 1860 . La forte représentation commerciale reflète en partie la spécialisation de la vieille ville. Il existe donc une adéquation assez prononcée aux lignes de force socio-économiques montréalaises.

Les confréries ont accueilli dans leur rang les représentants de la bourgeoisie francophone dominante avec toute sa diversité économique. Il s'agissait pour le clergé de ne pas les perdre au profit des "sociétés secrètes"', mais aussi pour ces laïss de donner l'exemple et de contribuer à l'encadrement social d'une masse ouvrière en formation. Très majoritairement, les congréganistes et les époux des dames de la Sainte-Famille possédaient leurs moyens de production ou exerçaient une profession honorable. Seule la Bonne-Mort a connu un recrutement plus modeste, caractère qui a dû être renforcé par la présence de célibataires de sexe féminin à la situation précaire en milieu urbain.

\section{La réorganisation et le renforcement de la discipline intérieure}

Toutes les confréries de dévotion instaurées en Nouvelle-France ont supporté le contrôle strict du clergé selon les orientations du Concile de Trente. Les clercs furent, dans la majorité des cas, les initiateurs de ces associations et pas une d'entre elles ne pouvait se réunir en l'absence du directeur ecclésiastique. La tenue des registres par ce dernier à Montréal en dit long sur son rôle, même si elle a eu l'avantage pour l'historien de préserver ces documents. Les laïcs prenaient part aux décisions concernant la vie de la confrérie par le biais d'officiers et de conseillers qu'ils avaient en

9 Jacques Grisé, Les conciles provinciaux de Québec et l'Église canadienne (1851-1886), Montréal, Fides, 1979. 
partie cooptés et élus. L'analyse des coutumiers et des procès-verbaux d'assemblées permet de mettre en évidence les changements d'équilibre entre les pouvoirs laïques et ecclésiastiques entre 1800 et 1850.

Durant cette période, les directeurs sont retournés aux règles en les appliquant plus strictement et en élaborant de nouveaux statuts. À la BonneMort et à la Sainte-Famille, ils ont à la fois remis de l'ordre et limité l'initiative laïque. Un coutumier, rédigé en 1834 par M. Léonard et inclus dans un livre de comptes, indique le renforcement du pouvoir du directeur de la Bonne-Mōort dont ie conseil perd iouie latitude: "quianand le Dircctcuir juge à propos d'assembler le Conseil, (ce qu'il doit faire le moins souvent que possible), il priera les conseillers de passer à la sacristie...» ${ }^{10}$ L'absence de registres rédigés par les laïcs dont c'était la tâche, comme le secrétairetrésorier, depuis la création de la confrérie, indique que les confrères n'ont jamais bénéficié de tous les pouvoirs que leur accordaient les statuts originels. Le directeur, responsable des dépenses, se contente de faire examiner les livres annuellement par le conseil. "Le directeur de la confrérie a soin de marquer exactement les services que la confrérie fait chanter pendant l'année, il les paye comme il est marqué sur le tarif ci dessus lc payement se fait avec l'argent du coffre de la confrérie, vers la mi-octobre, époque a-laquelle il rend compte des recettes et dépenses au conseil» ${ }^{11}$. Au XIXe siècle, un nouveau pas est franchi qui entraîne la confrérie vers un mouvement de dévotion peu structuré comme le Scapulaire.

Lorsque M. Sattin prit la tête de la Sainte-Famille en 1825, il dut se référer constamment aux statuts négligés depuis plusieurs années afin de rétablir l'assiduité aux assemblées et le respect du cérémonial. Son successeur, M. Roupe, suivit la même voie durant ses 20 années de direction (18341854), posant les jalons d'une autorité restaurée dont usera M. Martineau, dans la seconde moitié du siècle. Les consœurs y perdirent en prestige et en pouvoir. En 1846, la première dame de la confrérie doit reprendre le titre de première assistante et laisser celui de supérieure à la Vierge, car depuis 1724, les listes d'élection s'étaient éloignées des statuts qui prévoyaient que: «La Sainte Vierge sera reconnuë pour Supérieure, et la première assistante en fera les fonctions, avec subordination et soubs la conduite de celuy qui y présidera " ${ }^{12}$. La première atteinte au conseil est portée en 1826 lorsque les sacristines en sont exclues; toutefois le nombre de conseillères se maintient encore et les directeurs les convoquent régulièrement chaque mois contrairement au deuxième XIXe siècle. De plus, le mode de scrutin demeure

10 Cahier des recettes et dépenses de la confrérie de la Bonne-Mort depuis le 10 décembre 1834, ACSSM, S. 35, 16. 7.

11 Ibid.

12 La solide dévotion à la très sainte famille, de jesus, marie et joseph avec un catéchisme qui enseigne à pratiquer leurs vertus, Paris, Florentin Lambert, 1675, p. 38. 
inchangé, mêlant cooptation et élection pour choisir de nouvelles représentantes, suivant une rotation bien respectée. Avec M. Roupe, le directeur de la Sainte-Famille s'occupe de la sélection préliminaire des postulantes, en exigeant une entrevue avec elles avant la soumission de leur candidature au conseil. Le 24 novembre 1851, il lançait en assemblée cet «avis à celles qui se présentent pour la Sainte-Famille de venir m'en parler elles-mêmes» ${ }^{13}$, le conseil n'ayant plus qu'à entériner ce choix. Les prémices de la nouvelle répartition des pouvoirs apparaissent donc dans cette période de reprise en main par le clergé des confréries de dévotion, et tout particulièrement pour celles recrutant exclusivement ou essentiellement des femmes.

La Congrégation des hommes a connu une évolution inverse à la même époque en laissant plus de pouvoir aux laïcs. Nous disposons de documents abondants et riches pour en saisir la portée: un coutumier rédigé (ou recopié) par M. Chicoisneau entre 1805 et 1814 et qui constitue le document statutaire le plus ancien, conservé dans les archives malmenées de l'association; de nouveaux règlements élaborés en 1850 et approuvés par $\mathrm{M}^{\mathrm{gr}}$ Bourget le 8 décembre 1851 ; enfin toutes les décisions prises en conseil figurant dans les procès-verbaux qui ont tous été conservés au XIXe siècle. La participation des laïcs a toujours été plus grande à la Congrégation que dans les autres confréries étudiées. C'est ainsi que le trésorier, au moins depuis 1703, tenait régulièrement les comptes qu'il faisait vérifier par le conseil et le directeur, une fois l'an. À partir de 1830, ce conseil se scinde en un "grand" et un "petit" conseils, ce dernier servant d'exécutif à la Congrégation, pour accroître l'efficacité décisionnelle. De nombre variable, le grand conseil représentait, en 1830, le quart des congréganistes ayant versé leur cotisation annuelle et nouvellement reçus. De plus, toute décision majeure faisait l'objet d'une assemblée générale où elle était débattue et votée. En 1843, le petit conseil ajoute à ses prérogatives celle d'être consulté sur les demandes des futurs approbanistes, en plus de statuer sur les admissions elles-mêmes.

La même année, certaines décisions laissent présager un possible affaiblissement de la représentation laïque, lorsque les suppléants des grandes charges (seconds trésorier, secrétaire, maître des approbanistes) se voient écartés des conseils. En novembre, le système électoral est modifié avec la suppression de l'élection du préfet: le premier assistant devenant automatiquement préfet et le second remplaçant le premier. C'était courcircuiter l'élection qui se reportait sur le second assistant, en suivant toutefois la procédure habituelle: choix de trois candidats par le petit conseil et élection par l'ensemble des congréganistes. Le pouvoir, accordé à ces derniers et assez

13 Cahier des assemblées de la Sainte-Famille, 1848-1864, (désormais CASF), p. 57, 24 novembre 1851, ACSSM, carton 167. 
bien respecté par le clergé, explique le succès de cette confrérie auprès des notables montréalais.

Le retour aux règles et l'édition de nouveaux statuts ont incité les directeurs à recourir à de nouveaux moyens de contrôle de leur bonne application. C'est ainsi que d'anciens offices à la Congrégation et à la SainteFamille ont été réorientés vers la surveillance interne. Au siècle précédent, les visiteuses et préfets de quartier avaient été désignés pour venir en aide aux consœurs et confrères de leur voisinage. Les usages de $\mathrm{M}$. Chicoisneau faisaient référence, au début du siẹcle, aux visiteurs des malades dont la fonction s'explique clairement par le titre. Ensuite, M. Roupe qui a aussi dirigé la Congrégation parle de visiteurs, de zélateurs ou bien encore de préfets de quartier, dont les effectifs augmentent à mesure que la ville grandit. En 1831, s'affirme leur contrôle sur les congréganistes et vingt ans plus tard, le nouveau règlement relègue au second plan leur devoir de visiter les malades, d'annoncer les décès et de faire la charité: «Leur office est de veiller sur les confrères domiciliés en leur quartier, de les avertir charitablement, lorsqu'ils se négligent, ou qu'ils font quelque chose de répréhensible, de donner avis au Préfet des abus qui peuvent s'introduire dans la Congrégation ${ }^{14}$. La Sainte-Famille a connu une évolution semblable. Après une absence de nomination à cette charge entre 1788 et 1835 , en raison de la faiblesse des effectifs, M. Sattin fit adopter le principe de sa restauration en 1829 , lors d'une assemblée générale, et l'application revint à son successeur en 1835. Les dames de quartier se doivent désormais de comptabiliser les absences aux assemblées:

Je prie les Dames de quartier de se placer dans la chapelle comme je les en ai priées de manière à pouvoir s'assurer si celles de leur arrondissement sont présentes, afin de savoir la cause de leur absence, d'avertir charitablement les négligentes, et de m'en avertir moi-même si leur avertissement ne suffit pas. ${ }^{15}$

Deux ans plus tard, il ne s'agit plus de vérifier simplement l'assiduité mais de contrôler la conduite des consœurs dans le quartier:

Il est bon que les Dames de quartier aient par devant elles une liste des consœurs qui sont dans leur quartier respectif afin de les connoitre, de les visiter, de voire à chaque assemblée s'il en manque quelqu'une afin de les en avertir ou m'en prévenir et de s'informer de la vie et conduite de celles qu'elles ont dans leur arrondissement et de m'instruire de ce qu'elles peuvent en apprendre. ${ }^{16}$

Leur rôle devenait essentiel pendant les vacances de la confrérie, si dangereuses aux yeux des directeurs, et pendant lesquelles elles prenaient le relais des assemblées dans le contrôle des consœurs. Des directives morales furent en effet transmises à ces assemblées par les directeurs de la SainteFamille, surtout à partir des années 1830 , et se multiplièrent dans la

14 Manuel de piété à l'usage des Congréganistes de la Sainte Vierge de Ville-Marie, Montréal, J. B. Rolland et Fils, 1882, p. 332.

15 CASF, 1825-1848, p. 128, 7 juillet 1835, ACSSM, carton 167.

16 Ibid, p. 157,8 août 1837 . 
décennie suivante où de nouvelles pratiques dévotionnelles eurent pour but, par exemple, de canaliser les débordements festifs du carnaval. Dès son établissement à la Providence, en 1848, par $\mathrm{M}^{\mathrm{g}}$ Bourget, les exercices publics du Carnaval furent sanctifiés par le pieux souvenir des Douleurs de Marie. Ils s'agissait de faire passer le goût des réjouissances en faisant méditer, un mois durant, la vie douloureuse de la Vierge de l'Annonciation jusqu'à la sépulture de son fils.

Les confréries de dévotion montréalaises ont donc été l'objet d'une reprise en main antérieure à 1840 qui s'est traduite par un renforcement du pouvoir ecclésiastique et du contrôle des membres laïques. Toutefois, ces réorientations se confirmeront surtout dans la seconde moitié du siècle, avec des résultats parfois très négatifs, comme la délation systématique et pas toujours fondée, qui a brisé le climat confraternel à la Congrégation et à la Sainte-Famille.

\section{L'approfondissement et l'augmentation des exercices confraternels}

Outre la discipline, les Sulpiciens ont voulu restaurer les confréries comme écoles de piété, à la fois sur le plan individuel et collectif. Le retour des laïcs les encourageait dans cette voie afin d'éviter tout égarement de leur part.

Les cahiers des directeurs de la Sainte-Famille permettent d'évaluer le degré de leurs nouvelles exigences. Depuis les débuts de la confrérie, les pratiques privées valorisaient l'oraison mentale, qui, avec le chapelet, constituait les deux recommandations journalières essentielles. Le catéchisme qui accompagnait le manuel de la confrérie dans sa première édition de 1675 lui consacrait un chapitre complet. Pourtant, elle n'a pas été recommandée régulièrement par les directeurs. Au XIX' siècle, $\mathrm{M}$. Roupe sera le premier à en faire la promotion, encore ne le fait-il pas au début de son mandat, mais à partir de 1844. Il l'encadre d'une méthode pour éviter les risques de déviation, certainement soupesés dans une société dominée par les protestants.

En incitant la piété privée, le clergé voulait aussi créer un climat favorable au culte familial. Aussi, les dames de la Sainte-Famille furent-elles encouragées à établir un autel domestique autour duquel se réuniraient leurs proches pour réciter le chapelet. Les directeurs leur remettaient à cet effet, dès leur réception, une gravure représentant le saint modèle. À la fin du XVIIIe siècle, la traditionnelle remise de l'image a disparu et $\mathrm{M}$. Sattin la rétablit en 1825 dès son arrivée:

Je me suis arrangé, dit-il aux dames, avec les sœurs de l'Hôpital général pour en faire tirer une centaine de copies qui seront enluminées et ensuite encadrées sous 
des vitres, afin qu'elles servent d'ornements pour les appartements où elles seront placées ${ }^{17}$.

L'oraison devait s'alimenter de lectures spirituelles, à commencer par le manuel de la confrérie pour la Sainte-Famille.

La Congrégation, formée de lisants, fut précurseur en ce domaine, puisqu'elle fonda sa propre bibliothèque en 1835 , afin de détourner ses membres des mauvais livres. Dès l'entérinement du projet par le grand conseil, le 29 novembre 1835, l'orientation de l'organisme est décidée: «qu'il sêroit étábli immédiatement dans la sacristie de la chapelle... une bibliothèque composée d'un choix de livres dogmatiques et moraux pour l'usage des congréganistes et du public en général $"{ }^{18}$. Ce fut le premier pas vers une bibliothèque paroissiale qui prit tout d'abord la forme de l'Oeuvre des bons livres en $1844^{19}$. La réaction à la montée du prosélytisme protestant, aux idées voltairiennes et libérales, s'opère donc précocement afin d'éviter l'hémorragie des notables et permettre à ces derniers de nourrir leur piété de ces manuels qui inondent l'édition québécoise en ce siècle.

Les activités confraternelles collectives ont pris un nouvel essor, pendant cette période, confortant ainsi le sentiment d'appartenance et de fierté des consœurs et des confrères. Il s'agissait tout d'abord, pour les directeurs, d'assurer une bonne connaissance des règles de ces associations, afin d'éviter des engagements pris à la légère par des approbanistes peu enclins à se plier à des exercices nombreux et exigeants. En 1825, l'instructrice de la Sainte-Famille fait circuler le manuel entre les approbanistes qui devaient "communiquer de tems en tems, sur-tout dans les commencements, avec elle ${ }^{20}$. À la Congrégation, les futures recrues, outre leur assistance régulière aux offices, durent participer à des réunions supplémentaires «dans la chapelle tous les 15 jours à $1 \mathrm{~h}$ pm pour les exercer à la lecture du latin et du français et les instruire des principaux devoirs des congréganistes» ${ }^{21}$.

La participation régulière aux assemblées devait asseoir la cohésion du groupe et favoriser le progrès spirituel et moral. Or, l'absentéisme menaçait toujours des confréries exigeantes pour leurs associés. En 1837, le directeur de la Sainte-Famille ajoute la vénération des reliques aux fêtes des saints pour lesquels la confrérie en possédait quelques-unes. L'intérêt porté aux reliques faisait l'unanimité entre clercs et laïques et entraîna $M$. Roupe à les collectionner pour sa confrérie. Ainsi, en avril 1845, il obtint un reliquaire

17 CASF, 1825-1848, p. 10, 23 août 1825, ACSSM, carton 168 .

18 Registre des délibérations du conseil et des réceptions (désormais RDCR), 1835-1886, p. 1, Grand conseil 20 novembre 1835, AFNDM.

19 Marcel Lajeunesse, Les Sulpiciens et la vie culturelle à Montréal au XIXe siècle, Montréal, Fides, 1982, p. 220.

20 CASF, 1825-1848, p. 28, 19 septembre 1825, ACSSM, carton 167.

21 RDCR, 1835-1886, p. 38, 19 novembre 1842, AFNDM. 
de l'Hôtel-Dieu, contenant un morceau de la crèche, des sépulcres de Jésus et Marie, de l'habit de saint Joseph, des os de saint Jean-Baptiste, de sainte Anne et son époux ainsi que de Zacharie, Philomène et Janvier. Il systématise leur vénération «le tems de chaque assemblée, les jours de fêtes de la Ste Famille; aujourd'hui avant de les exposer, et après avoir donné une courte explication de ce que l'Église nous enseigne sur les Reliques, je vous les ferai d'abord vénérer "22. Le succès ne se fit pas attendre et $M$. Roupe reconnaissait qu'elles raffermissaient l'assiduité aux assemblées, mais l'engouement s'émoussa à force de répétition. Dès 1848 , le directeur devait espacer les expositions car «je crains, dit-il, de les exposer souvent, on ne marque pas de zèle à les y venir visiter ${ }^{23}$. La solennité renforcée des assemblées n'a certainement pas été étrangère à la suppression des vacances à la Sainte-Famille qui se fit à la demande des laïques, si nous en croyons le récit du directeur:

Depuis plusieurs années, plusieurs Dames de la Sainte famille avoient témoigné le désir que les assemblées... se continuassent pendant l'hyver, représentant que cinq mois de vacances ou d'interruption exposoient les consœurs à se rallentir de leur ferveur, ce projet avoit d'abord été rejetté comme une nouveauté, toujours dangereuse dans les confrérie. ${ }^{24}$

Le redressement opéré par MM. Sattin et Roupe a influencé cette initiative, tout en témoignant de sa réussite.

Outre la solennisation des assemblées, les fêtes particulières des confréries ont pris de l'ampleur dans la première moitié du siècle. Ces célébrations permettaient de manifester devant la paroisse la vitalité de leur association et conforter le sentiment d'appartenance à un groupe privilégié si ce n'est d'élite. Toutefois, la pompe n'exclua pas un approfondissement de la signification de ces cérémonies. Pour mieux préparer les associés à la fête patronale de la Sainte-Famille et de la Congrégation, les directeurs organisaient un triduum ou une retraite auparavant. Pour la Congrégation, nous ne possédons qu'une seule mention en 1855 qui en précise les dispositions financières. M. Roupe introduisit la pratique à la Sainte-Famille dix ans plus tôt. Ces exercices n'étaient pas obligatoires mais visaient à faire de la solennité une véritable rénovation des âmes. Les consœurs "se recueilleront et se disposeront, par la prière, la méditation et autres exercices à la réception des sacremens, et tacheront de se renouveller dans la ferveur et l'accomplissement fidele de leurs saintes regles ${ }^{25}$. Les dames devaient se rendre par deux fois à l'église de l'Hôtel-Dieu, pour des exercices encadrant la journée de travail: «L'oraison en commun tous les matins à $6 \mathrm{~h}$ 1/2 pendant

22 CASF, 1825-1848, p. 210, 8 avril 1845, ACSSM, carton 167.

23 Ibid, p. 256, 27 juin 1848.

24 Registre de la confrérie des dames de la Sainte-Famille, volume 1, 30 novembre 1847, ACSSM, carton 167.

25 CASF, 1825-1848, p. 210, 8 avril 1845, ACSSM, carton 167. 
une demie heure, qui sera suivi de la sainte messe à laquelle elles assisteront, et le soir à $5 \mathrm{~h} 1 / 2$ une instruction suivie du salut ${ }^{26}$. Le matin, le directeur proposait des pratiques à mener dans la journée, "pour celles des Dames qui auront plus de loisir». La retraite annuelle entra dans la tradition de la confrérie, à une époque où le clergé en espérait beaucoup pour encadrer l'ensemble des paroissiens.

Les confréries montréalaises ont participé et donné l'exemple des cérémonies que désirait le clergé au XIX ${ }^{\mathrm{e}}$ siècle dans le contexte d'une religion qui se voulaii publiquuée êt visitule, suivant le modèle italien, et prosélyte, en milieu protestant. Les associés prisaient ces célébrations publiques et la Congrégation des hommes a beaucoup fait pour celles-ci. Dans les années 1850, la fête de l'Assomption est solennisée par une grand'messe avec vêpres le soir. Les congréganistes de Ville-Marie y invitaient les congrégations, réaffirmant ainsi la supériorité de la congrégation mère, qui accueillait ses filles dans une chapelle entièrement ornée de lys, symbole de la virginité, et de branches de sapin ${ }^{27}$. L'accentuation de l'aspect liturgique des assemblées et des fêtes transparaît dans la création de nouveaux offices: celui de modérateur de chant en 1850 et de maître des cérémonies un an plus tard. L'absence de renseignements détaillés pour la Bonne-Mort ne signifie pas qu'elle ait été exclue de cette orientation qui toucha aussi la Sainte-Famille. Les directeurs de celle-ci passaient, chaque année, une partie des assemblées précédant les deux grandes fêtes de la confrérie (Sainte-Famille et Fête-Dieu) à répéter les points essentiels du cérémonial. Ils appréciaient l'effort de décoration de la chapelle presqu'autant que la piété des dames: «La fête de la Ste-Famille m'a parue célébrer avec beaucoup de piété; la Décoration de l'Autel, les Ste Reliques bien illuminées et surtout bien entourées, depuis le matin jusqu'après les Vêpres»" 28 . Cette orientation s'accentua encore tout au long du second XIXe.

Mais dès 1848 , les confréries traditionnelles tinrent leur rang dans les grandes manifestations du renouveau religieux montréalais en participant activement, par exemple, au rétablissement du pèlerinage de Bon-Secours qu'elles intégrèrent à leurs dévotions. À partir de cette date, les dames de la Sainte-Famille s'en allèrent tous les ans, «les plus jeunes en avant, d'un pas ni trop vite, ni trop lent ${ }^{29}$, pour honorer la Vierge dès l'aube, marquant par ce geste le début du mois consacré à la mère de Dieu. Les congréganistes choisirent le même mois pour leur pèlerinage et inclurent celui-ci dans le règlement édité en 1882. Nous savons indirectement que la Bonne-Mort a

26 Ibid, p. 210.

27 Registre des délibérations du petit conseil, 10 août 1851, AFNDM.

28 CASF, 1848-1864, p. 112, 15 avril 1856, ACSSM, carton 168.

29 Ibid., p. 12, 29 mai 1848. 
participé à la restauration de la dévotion par les dons d'ornements liturgiques qu'elle fit au sanctuaire. En juin 1848, il lui en a coûté «Pour une chasuble rouge donnée à Bon secours le jour du pèlerinage $35 \mathrm{sh}$ " et elle devait à "mr Chalbos sept Louis pour un calice» ${ }^{30}$. Les deux autres confréries y contribuèrent de la même façon, dans les années suivantes; il s'agissait pour toutes de favoriser l'éclat du pèlerinage mais aussi de manifester leur existence dans la paroisse, non sans en retirer une certaine fierté.

Ainsi, les confréries de dévotion anciennes ont participé activement aux mutations religieuses qui ont secoué la société montréalaise et québécoise, et qui ont présidé à l'instauration du catholicisme tel que nous l'avons connu jusque dans les années 1950. Le retour des laïcs vers ces confréries a précédé le réveil religieux, lui fournissant des éléments dynamiques par la qualité de son recrutement. Les Sulpiciens ont restructuré les associations de recrutement spécialisé sexuellement avant le lancement des innovations de Mgr Bourget qui, au-delà des conflits avec ces Messieurs, a pu trouver des laïs ouverts aux nouvelles dévotions et prêts à se conformer aux exigences de celles-ci. Les directeurs invitèrent les associés à prendre part aux neuvaines, mois et différents mouvements de piété, instaurés dans les années 1840-60. Ils apprécièrent la présence épiscopale aux assemblées de la SainteFamille et de la Congrégation qui en retiraient un prestige accru, très favorable au maintien du recrutement. Les confréries de dévotion anciennes fournirent donc aussi un terrain d'entente aux deux pôles de l'autorité religieuse montréalaise, facilitant peut-être le succès des réformes et la réconciliation.

30 ACSSM, S. 35, 16.7. 
Confrérie des dames de la Sainte-Famille

$\exists$ Congrégation des hommes de Ville-Marie

$\varpi$ Confrérie de la Bonne-Mort

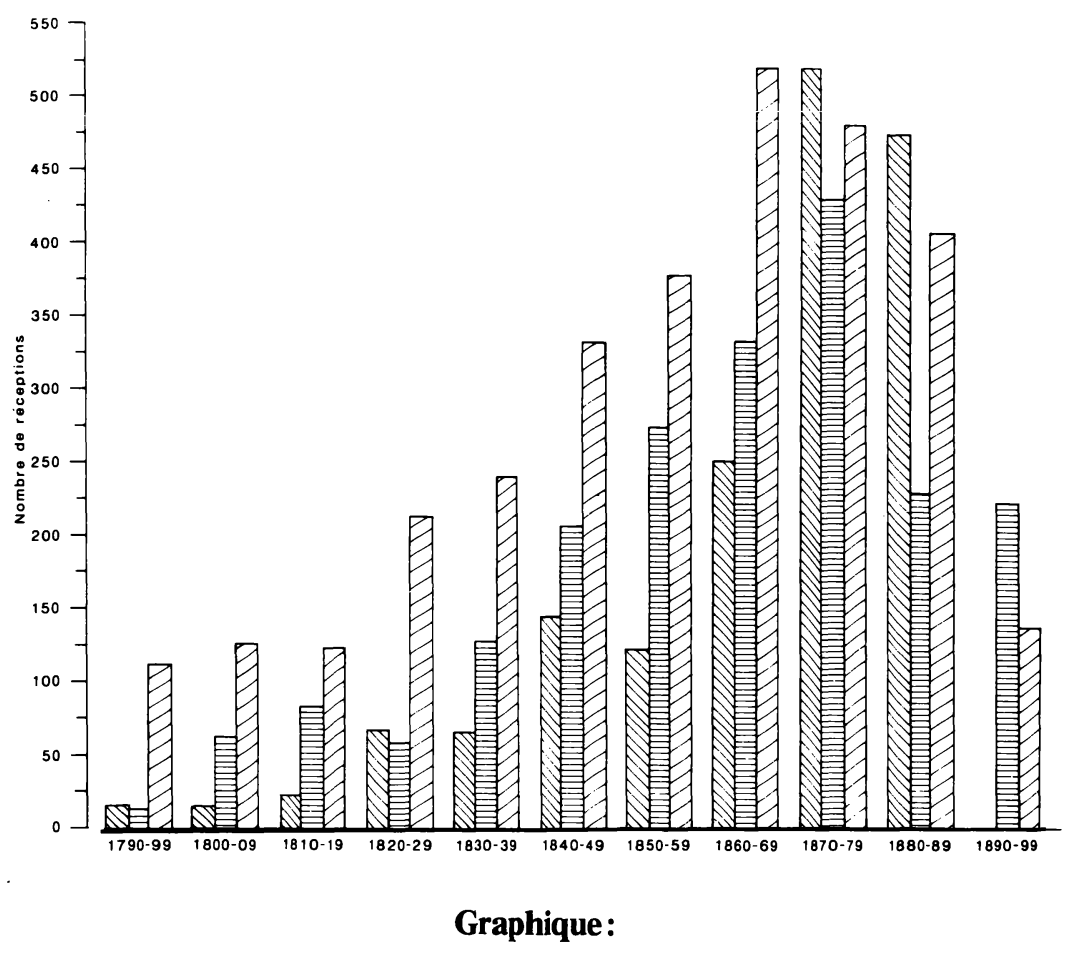

Évolution comparée du recrutement décennal 